\title{
Entanglement entropy of asymptotically flat non-extremal and extremal black holes with an island
}

\author{
Wontae Kim $^{1,2, a}$, Mungon Nam ${ }^{1,2, b}$ \\ ${ }^{1}$ Department of Physics, Sogang University, Seoul 04107, Republic of Korea \\ ${ }^{2}$ Center for Quantum Spacetime, Sogang University, Seoul 04107, Republic of Korea
}

Received: 13 July 2021 / Accepted: 22 September 2021 / Published online: 5 October 2021

(C) The Author(s) 2021

\begin{abstract}
The island rule for the entanglement entropy is applied to an eternal Reissner-Nordström black hole. The key ingredient is that the black hole is assumed to be in thermal equilibrium with a heat bath of an arbitrary temperature and so the generalized entropy is treated as being off-shell. Taking the on-shell condition to the off-shell generalized entropy, we find the generalized entropy and then obtain the entanglement entropy following the island rule. For the non-extremal black hole, the entanglement entropy grows linearly in time and can be saturated after the Page time as expected. The entanglement entropy also has a welldefined Schwarzschild limit. In the extremal black hole, the island prescription provides a logarithmically growing entanglement entropy in time and a constant entanglement entropy after the Page time. In the extremal black hole, the boundary of the island hits the curvature singularity where the semiclassical approximations appear invalid. To avoid encountering the curvature singularity, we apply this procedure to the Hayward black hole regular at the origin. Consequently, the presence of the island in extremal black holes can provide a finite entanglement entropy, which might imply non-trivial vacuum configurations of extremal black holes.
\end{abstract}

\section{Introduction}

Since Hawking's discovery that black hole radiates [1], the information loss paradox has been one of the most intriguing issues in quantum gravity [2]. If unitarity in quantum mechanics is advocated, then information of black holes should be conserved during the process of evaporation, of course, the word "unitarity" can be used in different meaning [3]. From the viewpoint of the entanglement entropy of thermal radiation in evaporating black holes, it starts with the

\footnotetext{
a e-mail:wtkim@sogang.ac.kr (corresponding author)

b e-mail: clrchr0909@sogang.ac.kr
}

zero entropy and reaches a peak at the Page time and finally ends up with the zero entropy [4]. After the Page time, the entanglement entropy of radiation follows the curve of the decreasing thermal entropy of black holes.

On the other hand, a holographic derivation of the entanglement entropy was proposed from AdS/CFT correspondence $[5,6]$, and quantum corrections to the entanglement entropy were also studied in Refs. [7,8]. Then, the entanglement entropy was calculated at arbitrary orders in the bulk Planck constant by using the quantum extremal surface which extremizes the generalized entropy of the sum of area and bulk entanglement entropy [9]. In general, a density matrix at a region outside of black hole is obtained by a partial trace over the state in complementary region. The states inside a surface so called the island region [10-13] need to be removed from the states which are traced out. The emergence of islands was also shown by using the replica trick $[14,15]$. Thus, the entanglement entropy at some region of Hawking radiation $R$ should be represented by the union of $R$ and its island region $I$. According to the prescription of minimal quantum extremal surface [9], the fine-grained entanglement entropy of the Hawking radiation is proposed as [13]

$$
S=\min \left\{\operatorname{ext} S_{\text {gen }}\right\}=\min \left\{\operatorname{ext}\left[\frac{\mathcal{A}(\partial I)}{4 G_{N}}+S_{\text {matter }}(R \cup I)\right]\right\},
$$

where $S_{\text {gen }}$ is the generalized entropy and $\mathcal{A}(\partial I)$ is the area of the boundary of island $I$. The matter part of the entanglement entropy in Eq. (1) includes the UV divergence proportional to the island area, subject to a short distance proper cut-off scale [16,17], and thus, the Newton constant $G_{N}$ must be renormalized [18]. The prescription of minimal quantum extremal surface was initially applied to the two-dimensional AdS spacetime, and then it was applied to arbitrary dimensions [19] and an eternal Schwarzschild black hole [20] as 
well as various interesting black holes [21-25] with discussions on important role of gravitating bath [26-30].

In particular, for an extremal Reissner-Nordström black hole, the entropy was shown to vanish for an arbitrary temperature of a heat bath in the Euclidean action formalism [31]. In addition, it was proposed that the entropy can vanish when taking a vanishing temperature by employing ' $t$ Hooft's approach [32] evaluating black hole entropy through a statistical-mechanical counting of states for a scalar field propagating outside the event horizon [33]. Of course, a nonzero entropy was also derived for a stringy extremal black hole by microscopic counting of degrees of freedom in sting theory without resort to mentioning the status of the temperature [34]. On the other hand, the entropy of extremal black holes turned out to be different from that of the near extremal limit [35]. In fact, in the Eddington-Finkelstein system, there is a discontinuity between the non-extremal black hole and the extremal one despite the well-defined metrics. The entropy of extremal black holes still remains as an intriguing one. Thus, it would be interesting to study the entanglement entropy of a black hole with inner and outer horizons in order to figure out how the presence of the island influences the entanglement entropy in extremal black holes.

Starting from a black hole in thermal equilibrium with a heat bath of an arbitrary temperature $\beta$, one can obtain the area law of the black hole entropy by taking as $\beta \rightarrow \beta_{H}$ where $\beta_{H}$ is the inverse temperature of the black hole $[32,33]$. In this respect, we suggest that a black hole with an island is also assumed to be in thermal equilibrium with a heat bath of an arbitrary temperature in such a way that at the interim stage the off-shell generalized entropy $S_{\text {gen }}(\kappa)$ can be constructed. Taking $\kappa \rightarrow \kappa_{H}$, we obtain the generalized entropy in Eq. (1) as

$S_{\mathrm{gen}}=\lim _{\kappa \rightarrow \kappa_{H}} S_{\mathrm{gen}}(\kappa)$,

where $\kappa=2 \pi T=2 \pi \beta^{-1}$ are off-shell quantities and $\kappa_{H}$ is the surface gravity at the horizon. This formula will be used in calculating the entanglement entropy for non-extremal and extremal black holes. This process is actually trivial for non-extremal black holes but crucial for extremal ones, which will be discussed in forthcoming sections. Along the line of Ref. [20] with the assumption (2), we first revisit the computation of the entanglement entropy in the nonextremal Reissner-Nordström black hole with an island and then obtain a slightly different form of the entanglement entropy from the previous result in Ref. [25]. In our case, the entanglement entropy reduces to that of the Schwarzschild black hole when the electric charge vanishes [20]. In the extremal Reissner-Nordström black hole of our main interest, we compute the entanglement entropy with and without an island. To avoid encountering the curvature singularity at the origin in the Reissner-Nordström black hole, we repeat the computation of the entanglement entropy for the regular Hayward black hole [36].

The organization of this paper is as follows. In Sect. 2, we will obtain the generalized entropy from the off-shell generalized entropy for non-extremal and extremal black holes with and without the island. In Sect. 3, we will calculate the entanglement entropy of the Reissner-Nordström black hole. In Sect. 4 , the process performed in Sect. 3 will be applied to the Hayward black hole. Finally, conclusion and discussion will be given in Sect. 5 .

\section{Generalized entropy}

We study the entanglement entropy of asymptotically flat spherically symmetric eternal black holes described by a length element

$\mathrm{d} s^{2}=-f(r) \mathrm{d} t^{2}+\frac{1}{f(r)} \mathrm{d} r^{2}+r^{2} \mathrm{~d} \Omega^{2}$,

where $f(r)$ is a metric function which has two horizons $r_{+}$ and $r_{-}$with $r_{+}>r_{-}$. The metric in terms of Kruskal coordinates is defined by

$\mathrm{d} s^{2}=-e^{2 \rho} \mathrm{d} X^{+} \mathrm{d} X^{-}+r^{2} \mathrm{~d} \Omega^{2}$,

where $\kappa X^{ \pm}= \pm e^{ \pm \kappa\left(t \pm r_{*}\right)}$ and $r_{*}$ is a radial tortoise coordinate defined by $r_{*}=\int^{r}(1 / f(r)) \mathrm{d} r$, and $e^{2 \rho}=$ $f(r) /\left(-\kappa^{2} X^{+} X^{-}\right)$. The Hawking temperature is calculated as

$T_{H}=\beta_{H}^{-1}=\frac{\kappa_{H}}{2 \pi}=\frac{f^{\prime}\left(r_{+}\right)}{4 \pi}$

which is now called the on-shell temperature.

For a finite matter part of the entanglement entropy in curved spacetime, we consider free massless scalar fields and make an assumption that the distance between the boundary surfaces is large compared to the scale of the size of the boundary surfaces so that only the s-wave contributes to the entropy [20]. Thus, from the two-dimensional conformal field theory, the matter part of the entanglement entropy is approximately given as

$S_{\text {matter }}=\frac{c}{3} \log d(x, y)$

where $c$ is a central charge for a two-dimensional massless scalar field and $d(x, y)$ is a distance between $x$ and $y$. In fact, the matter part of the entanglement entropy in the logarithmic correction also includes the proper UV cut-off scale $\epsilon$ [37], but it will be deleted as an additive constant. 


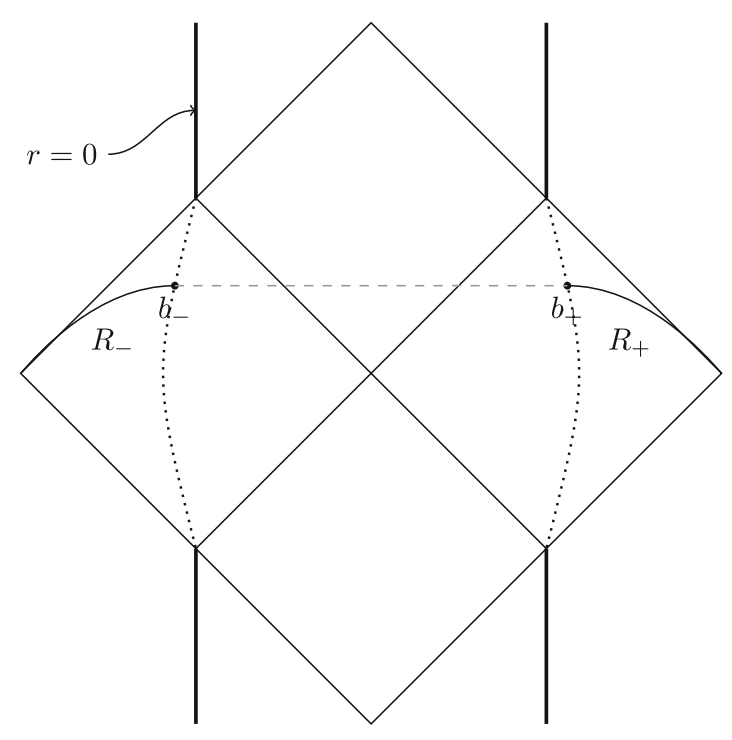

(a) Without island

Fig. 1 The Penrose diagrams of an eternal non-extremal black hole are presented without an island (left) and with an island (right). The region for the Hawking radiation $R$ is separated into two parts; $R_{+}$and $R_{-}$located in the right and left wedges, respectively. The boundaries

\subsection{Non-extremal black hole}

\subsubsection{Without island}

The matter part of the generalized entropy is defined by the entanglement region between the boundaries $b_{+}=\left(t_{b}, b\right)$ and $b_{-}=\left(-t_{b}+i \beta / 2, b\right)^{1}$ in Fig. 1 as

$S_{\text {matter }}=\frac{c}{3} \log d\left(b_{+}, b_{-}\right)$

where $d^{2}(x, y)=-\left(X^{+}(x)-X^{+}(y)\right)\left(X^{-}(x)-X^{-}(y)\right) e^{\rho(x)}$ $e^{\rho(y)}$. Note that the black hole is in thermal equilibrium with a heat bath of an arbitrary temperature $\beta^{-1}$. Using Eqs. (2) and (7), we have

$S_{\mathrm{gen}}=\lim _{\kappa \rightarrow \kappa_{H}} S_{\mathrm{gen}}(\kappa)=\frac{c}{6} \log \left(\frac{4 f(b)}{\kappa_{H}^{2}} \cosh ^{2} \kappa_{H} t_{b}\right) \approx \frac{c}{3} \kappa_{H} t_{b}$

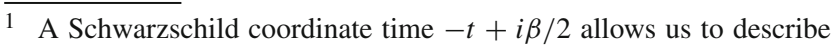
a mirror point located in the left wedge of the Penrose diagram, which means that $X^{ \pm}\left(x_{+}\right)=X^{\mp}\left(x_{-}\right)$in Kruskal coordinates where $x_{+}=$ $(t, r)$ in the right wedge and $x_{-}=(-t+i \beta / 2, r)$ in the left wedge. This fact can be found from the definition of Kruskal coordinates as

$$
\begin{aligned}
& \kappa X^{ \pm}\left(x_{-}\right)= \pm e^{ \pm \kappa\left(-t+\frac{i \beta}{2} \pm r_{*}\right)}= \pm e^{ \pm \kappa\left(-t+\frac{i \pi}{\kappa} \pm r_{*}\right)} \\
& = \pm e^{i \pi} e^{ \pm \kappa\left(-t \pm r_{*}\right)}=\mp e^{\mp \kappa\left(t \mp r_{*}\right)}=\kappa X^{\mp}\left(x_{+}\right),
\end{aligned}
$$

which results in $X^{ \pm}\left(x_{+}\right)=X^{\mp}\left(x_{-}\right)$.
}

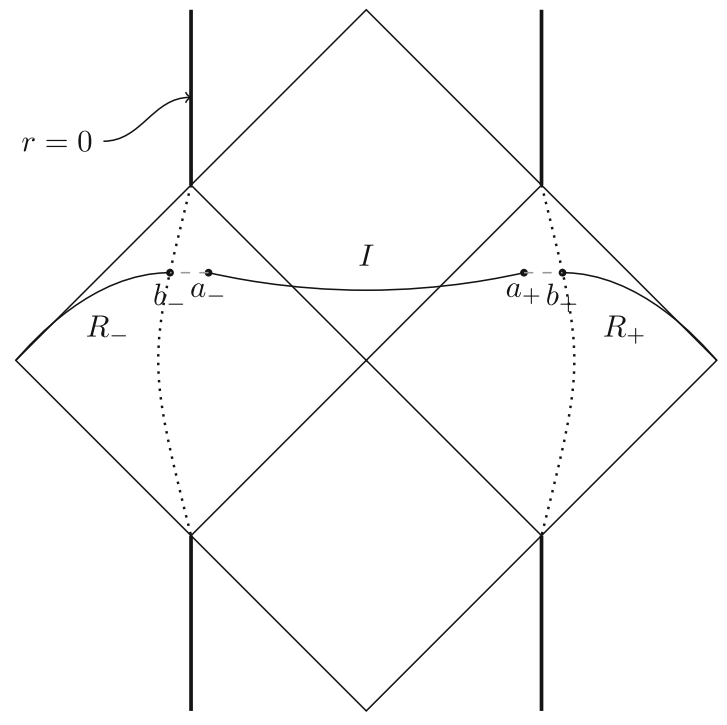

(b) With island

of $R_{+}$and $R_{-}$are indicated as $b_{+}$and $b_{-}$, respectively. The island extends between the two wedges and its boundaries are at $a_{+}$and $a_{-}$. In the Reissner-Nordström black hole, the curvature is singular at $r=0$ whereas it is finite there in the Hayward black hole

in the late time approximation of $t_{b} \gg 1 / \kappa_{H}$. This implies that the entanglement entropy is increasing with respect to time $t_{b}$ so that this entropy becomes much larger than the black hole entropy expected in Ref. [2]. Note that Eq. (8) in the late time approximation is valid only for non-extremal black holes.

\subsubsection{With island}

The collection of disjoint intervals is defined as $R \cup I=$ $R_{-} \cup I \cup R_{+}$, where the finite boundaries of $R_{+}$and $R_{-}$ are denoted by $b_{+}$and $b_{-}$, respectively. The island extends between the two radiation wedges and its boundaries are at $a_{+}$and $a_{-}$depicted in Fig. 1.

Let us now introduce a single island in the calculation of the entanglement entropy. The matter part with an island whose boundaries located at $a_{+}=\left(t_{a}, a\right)$ and $a_{-}=\left(-t_{a}+\right.$ $i \beta / 2, a)$ is given by

$$
S_{\text {matter }}=\frac{c}{3} \log \frac{d\left(a_{+}, a_{-}\right) d\left(b_{+}, b_{-}\right) d\left(a_{+}, b_{+}\right) d\left(a_{-}, b_{-}\right)}{d\left(a_{+}, b_{-}\right) d\left(a_{-}, b_{+}\right)} .
$$

The boundary of $R$ is assumed to be far away from the outer horizon $r_{+}$, which makes the s-wave approximation valid, and the island extends vicinity of the outside of the event horizon. From Eqs. (1), (2) and (9), the generalized entropy for the metric (3) is calculated as 
Fig. 2 The Penrose diagrams of an eternal extremal black hole are presented without an island (left) and with an island (right). The region for the Hawking radiation $R$ is located in the right wedge. The boundary of $R$ is denoted by $b_{+}$. In a, a reference point $b_{0}$ is introduced at $r=0$. In $\mathbf{b}$, the island extends between $r=0$ and $a_{+}$just outside the horizon. In the Reissner-Nordström black hole, the curvature is singular at $r=0$ whereas it is finite there in the Hayward black hole

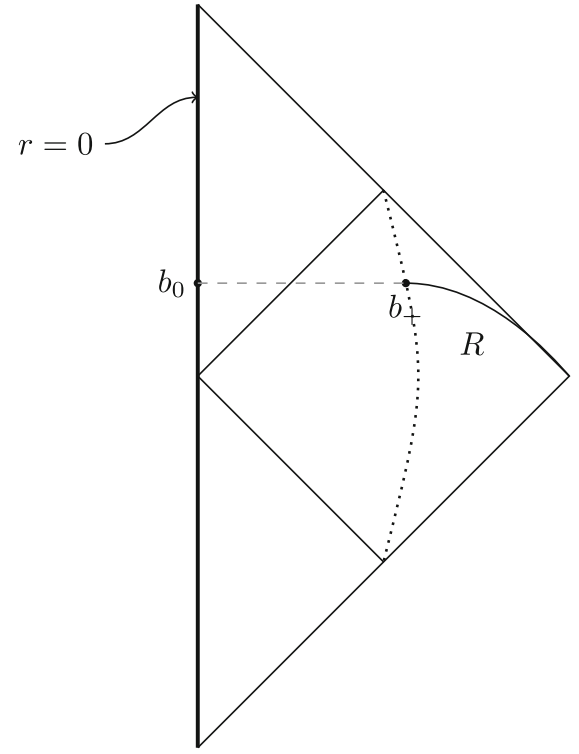

(a) Without island

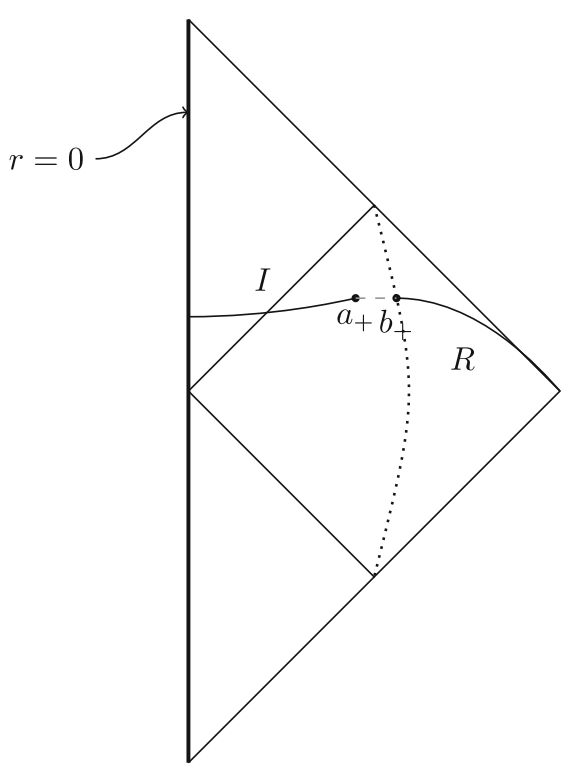

(b) With island

$$
\begin{aligned}
S_{\text {gen }}= & \lim _{\kappa \rightarrow \kappa_{H}} S_{\text {gen }}(\kappa) \\
= & \frac{2 \pi a^{2}}{G_{N}}+\frac{c}{6} \log \left(\frac{16 f(a) f(b)}{\kappa_{H}^{4}} \cosh ^{2} \kappa_{H} t_{a} \cosh ^{2} \kappa_{H} t_{b}\right) \\
& +\frac{c}{3} \log \left|\frac{\cosh \kappa_{H}\left(r_{*}(a)-r_{*}(b)\right)-\cosh \kappa_{H}\left(t_{a}-t_{b}\right)}{\cosh \kappa_{H}\left(r_{*}(a)-r_{*}(b)\right)+\cosh \kappa_{H}\left(t_{a}+t_{b}\right)}\right| .
\end{aligned}
$$

In fact, there is no distinction between the on-shell and the off-shell formalism in non-extremal black holes.

One might wonder in Eqs. (7) and (8) why the nonextremal black hole is surrounded by a heat bath of an arbitrary temperature. Usually non-extremal black holes evaporate in a lower-temperature bath and gain energy in a bath at higher temperatures. In this work, we assume eternal black holes possessing an arbitrary temperature as an off-shell temperature different from the on-shell temperature defined by the Hawking temperature. Of course, the heat bath also has the same arbitrary temperature as the black hole temperature. Therefore, the black hole is in thermal equilibrium with the heat bath and so it does not evaporate. After calculations, we take the on-shell limit as $\beta \rightarrow \beta_{H}$, i.e., $\kappa \rightarrow \kappa_{H}$. This kind of limiting process in thermal equilibrium has been used in calculating thermodynamic quantities in non-extremal and extremal black holes [32,33].

\subsection{Extremal black hole}

\subsubsection{Without island}

Let us take a spacelike surface which ends at a reference point $b_{0}=\left(t_{0}, 0\right)$ in Fig. 2 . Then, the entanglement entropy can be calculated as

$$
S_{\text {matter }}=\frac{c}{3} \log d\left(b_{+}, b_{0}\right),
$$

and so one can obtain

$$
\begin{aligned}
S_{\text {gen }}= & \lim _{\kappa \rightarrow 0} S_{\text {gen }}(\kappa)=\frac{c}{12} \log \\
& \times\left(f(0) f(b)\left[\left(r_{*}(b)-r_{*}(0)\right)^{2}-\left(t_{b}-t_{0}\right)^{2}\right]^{2}\right) .
\end{aligned}
$$

Note that $f(0)$ is singular for the Reissner-Nordström black hole so that the generalized entropy becomes infinite. The infinite entropy in the extremal Reissner-Nordström black hole is also a consequence of the infinite proper distance of $\left[b_{0}, b_{+}\right]$.

\subsubsection{With island}

The entanglement-entropy formula for a single interval $R \cup I$ is given by

$S_{\text {matter }}=\frac{c}{3} \log d\left(a_{+}, b_{+}\right)$.

In Fig. 2, the boundary of $R$ is assumed to be far away from the horizon $r_{+}=r_{-}=r_{h}$. From Eqs. (2) and (13), the offshell generalized entropy for the classical metric (3) for the extremal black hole is calculated as

$$
\begin{aligned}
S_{\text {gen }}(\kappa)= & \frac{\pi a^{2}}{G_{N}}+\frac{c}{12} \log \left(\frac{4 f(a) f(b)}{\kappa^{4}}\right) \\
& +\frac{c}{6} \log \left|\cosh \kappa\left(r_{*}(a)-r_{*}(b)\right)-\cosh \kappa\left(t_{a}-t_{b}\right)\right| .
\end{aligned}
$$


Taking the limit $\kappa \rightarrow 0$ in Eq. (14), we have a finite value of the generalized entropy as

$$
\begin{aligned}
S_{\text {gen }}= & \lim _{\kappa \rightarrow 0} S_{\text {gen }}(\kappa)=\frac{\pi a^{2}}{G_{N}}+\frac{c}{12} \log \\
& \times\left(f(a) f(b)\left[\left(r_{*}(a)-r_{*}(b)\right)^{2}-\left(t_{a}-t_{b}\right)^{2}\right]^{2}\right) .
\end{aligned}
$$

Note that the divergences from the second and third terms in Eq. (14) exactly cancel out.

In the next consecutive sections, we will apply the generalized entropy formulas to the Reissner-Nordström black hole and the Hayward black hole.

\section{Entanglement entropy of Reissner-Nordström black hole}

The length element of the Reissner-Nordström black hole is given as

$$
\begin{aligned}
\mathrm{d} s^{2}= & -\left(1-\frac{2 G_{N} M}{r}+\frac{G_{N} Q^{2}}{r^{2}}\right) \mathrm{d} t^{2} \\
& +\left(1-\frac{2 G_{N} M}{r}+\frac{G_{N} Q^{2}}{r^{2}}\right)^{-1} \mathrm{~d} r^{2}+r^{2} \mathrm{~d} \Omega^{2},
\end{aligned}
$$

where $M$ is the black-hole mass and $Q$ is the electric charge of the black hole. The non-extremal black hole has two horizons $r_{ \pm}=G_{N}\left(M \pm \sqrt{M^{2}-Q^{2}}\right)$. If the black hole is extremal: $M=Q$, then $r_{+}=r_{-}$and the horizon reduces to $r_{h}=$ $G_{N} M$. The Reissner-Nordström black hole has a curvature singularity at $r=0$.

\subsection{Non-extremal Reissner-Nordström black hole}

We calculate the entanglement entropy of the non-extremal Reissner-Nordström black hole described by the metric function

$f(r)=\frac{\left(r-r_{+}\right)\left(r-r_{-}\right)}{r^{2}}$.

In the absence of the island, the entanglement entropy is nothing but Eq. (8).

Let us derive the entanglement entropy of the nonextremal Reissner-Nordström black hole with an island. In the late time approximation of $t_{a}+t_{b} \rightarrow \infty$ [20], the generalized entropy (10) for the metric (17) is extremized with respect to $t_{a}$ as

$$
\frac{\partial S_{\mathrm{gen}}}{\partial t_{a}}=\frac{c \kappa_{H} \sinh \kappa_{H}\left(t_{a}-t_{b}\right)}{3\left[\cosh \kappa_{H}\left(t_{a}-t_{b}\right)-\cosh \kappa_{H}\left(r_{*}(a)-r_{*}(b)\right)\right]}=0,
$$

which simply yields $t_{a}=t_{b}$. In Eq. (18), the surface gravity $\kappa_{H}$ is defined by

$\kappa_{H}=\frac{r_{+}-r_{-}}{2 r_{+}^{2}}$,

and the boundaries of the island and the radiation region in terms of tortoise coordinates are also given by

$$
\begin{aligned}
r_{*}(a)= & r_{+}+\frac{r_{+}^{2}}{r_{+}-r_{-}} \log \left(\frac{a-r_{+}}{r_{+}}\right) \\
r_{*}(b)= & b+\frac{r_{+}^{2}}{r_{+}-r_{-}} \log \left(\frac{b-r_{+}}{r_{+}}\right)-\frac{r_{-}^{2}}{r_{+}-r_{-}} \\
& \times \log \left(\frac{b-r_{-}}{r_{+}}\right) .
\end{aligned}
$$

Next, the derivative of the generalized entropy (10) with respect to $a$ is obtained as

$$
\begin{aligned}
\frac{\partial S_{\mathrm{gen}}}{\partial a} & =\frac{4 \pi a}{G_{N}}+\frac{c}{6 f(a)}\left[f^{\prime}(a)-2 \kappa_{H}-4 \kappa_{H} e^{\kappa_{H}\left(r_{*}(a)-r_{*}(b)\right)}\right] \\
& =0
\end{aligned}
$$

by using the fact that $t_{a}=t_{b}, t_{a}+t_{b} \rightarrow \infty$, and $a \sim r_{+}$. From Eq. (22), the boundary of the island can approximately be determined as

$a=r_{+}+\frac{\left(c G_{N}\right)^{2}}{144 \pi^{2} r_{+}^{3}} e^{2 \kappa_{H}\left(r_{+}-r_{*}(b)\right)}+\mathcal{O}\left(G_{N}^{3}\right)$.

From Eq. (1), the entanglement entropy of the nonextremal Reissner-Nordström black hole with an island is obtained as

$$
\begin{aligned}
S_{\mathrm{I}}= & \frac{2 \pi r_{+}^{2}}{G_{N}}+\frac{c}{6}\left[\log \left(\frac{16 r_{+}^{6}\left(b-r_{+}\right)^{2}\left(b-r_{-}\right)}{b^{2}\left(r_{+}-r_{-}\right)^{3}}\right)\right. \\
& \left.-\frac{r_{-}^{2}}{r_{+}^{2}} \log \left(\frac{b-r_{-}}{r_{+}}\right)+\frac{r_{+}-r_{-}}{r_{+}^{2}}\left(b-r_{+}\right)\right] .
\end{aligned}
$$

The expression of the entanglement entropy (24) looks different from that in Ref. [25] in the sense that the Schwarzschild limit appears to be different. For $r_{-}=0$, Eq. (24) exactly reduces to the entanglement entropy of the Hawking radiation in the Schwarzschild black hole [20],

$S_{\mathrm{I}}=\frac{2 \pi r_{h}^{2}}{G_{N}}+\frac{c}{6}\left[\log \left(\frac{16 r_{h}^{3}\left(b-r_{h}\right)^{2}}{b}\right)+\frac{b-r_{h}}{r_{h}}\right]$.

In the non-extremal Reissner-Nordström black hole, the Page time can be estimated at which the growing entanglement entropy (8) changes into the constant entanglement entropy (24). The Page time is the same form as that of the 
Schwarzschild black hole in Ref. [20], which is almost universal,

$t_{\text {Page }}=\frac{6 \pi r_{+}^{2}}{\kappa_{H} c G_{N}}=\frac{3 S_{\mathrm{BH}}}{\pi c T_{\mathrm{H}}}$,

where $S_{\mathrm{BH}}=\left(\pi r_{+}^{2}\right) / G_{N}$ is the Bekenstein-Hawking entropy. The Page time is getting longer when the black-hole mass is close to the electric charge.

\subsection{Extremal Reissner-Nordström black hole}

Let us calculate the entanglement entropy of the extremal Reissner-Nordström black hole described by the metric function as

$f(r)=\frac{\left(r-r_{h}\right)^{2}}{r^{2}}$.

We investigate the entanglement entropy of the extremal Reissner-Nordström black hole with an island. Extremizing the generalized entropy (15) for the metric (27) with respect to $t_{a}$ gives

$\frac{\partial S_{\text {gen }}}{\partial t_{a}}=\frac{c\left(t_{a}-t_{b}\right)}{3\left[\left(t_{a}-t_{b}\right)^{2}-\left(r_{*}(a)-r_{*}(b)\right)^{2}\right]}=0$,

where $r_{*}(a)$ and $r_{*}(b)$ are obtained through Eq. (27) as

$r_{*}(a)=r_{h}-\frac{r_{h}^{2}}{a-r_{h}}$,

$r_{*}(b)=b-\frac{r_{h}^{2}}{b-r_{h}}+2 r_{h} \log \left(\frac{b-r_{h}}{r_{h}}\right)$.

In this case, without resort to the late time approximation of $t_{a}+t_{b} \rightarrow \infty$, one can simply get $t_{a}=t_{b}$.

Next, the derivative of the generalized entropy (15) with respect to $a$ is found to be

$\frac{\partial S_{\mathrm{gen}}}{\partial a}=\frac{2 \pi a}{G_{N}}+\frac{c}{12 f(a)}\left(f^{\prime}(a)+\frac{4}{r_{*}(a)-r_{*}(b)}\right)=0$

within the approximation $a \sim r_{+}$. Hence, the boundary of the island can be determined as

$a=r_{h}+\frac{c G_{N}}{12 \pi r_{h}}+\frac{\left(c G_{N}\right)^{2}\left(7 r_{h}-2 r_{*}(b)\right)}{144 \pi^{2} r_{h}^{4}}+\mathcal{O}\left(G_{N}^{3}\right)$.

From Eq. (1), the entanglement entropy of the extremal Reissner-Nordström black hole with an island is finally obtained as

$S_{\mathrm{I}}=\frac{\pi r_{h}^{2}}{G_{N}}+\frac{c}{6} \log \left(\frac{12 \pi r_{h}^{4}\left(b-r_{h}\right)}{c G_{N} b}\right)$.
Note that the entanglement entropy (33) cannot be obtained from the continuous extremal limit of the non-extremal entanglement entropy (24) because the extremal case and the extremal limit are in essence different [35].

In Fig. 2, the island hits the boundary of the island and $f(0)$ is also divergent so that the semi-classical approximation in that region appears to be invalid. In this regard, we would repeat the computation of the entanglement entropy for a regular black hole in the next section.

\section{Entanglement entropy of Hayward black hole}

In this section, we study the entanglement entropy of the Hayward black hole described by the metric [36]

$$
\begin{aligned}
\mathrm{d} s^{2}= & -\left(1-\frac{2 G_{N} M r^{2}}{r^{3}+2 G_{N} M \ell^{2}}\right) \mathrm{d} t^{2} \\
& +\left(1-\frac{2 G_{N} M r^{2}}{r^{3}+2 G_{N} M \ell^{2}}\right)^{-1} \mathrm{~d} r^{2}+r^{2} \mathrm{~d} \Omega^{2},
\end{aligned}
$$

where $M$ is the black-hole mass and $\ell$ is the Hubble length. The metric is asymptotically flat such that it approaches the Schwarzschild metric as

$f(r) \sim 1-\frac{2 G_{N} M}{r}$ as $r \rightarrow \infty$.

Note that the metric behaves like the de Sitter space near the origin $r=0$ as

$f(r) \sim 1-\frac{r^{2}}{\ell^{2}} \quad$ as $\quad r \rightarrow 0$,

where the curvature is finite at the origin. Unlike the Reissner-Nordström black hole, the Hayward black hole is regular everywhere.

\subsection{Non-extremal Hayward black hole}

In Eq. (34), the metric function of the non-extremal Hayward black hole is neatly written as

$f(r)=\frac{\left(r-r_{+}\right)\left(r-r_{-}\right)\left(r-r_{0}\right)}{r^{3}-r_{+} r_{-} r_{0}}$,

where $r_{+}$and $r_{-}$are two positive roots satisfying

$r^{3}-2 G_{N} M r^{2}+2 G_{N} M \ell^{2}=0$,

and $r_{0}=2 G_{N} M-\left(r_{+}+r_{-}\right)$which is negative. If $M>$ $\left(3 \sqrt{3} / 4 G_{N}\right) \ell$, the black hole has two horizons: $r_{+}$and $r_{-}$ where $r_{+}>r_{-}$. For $r_{-}=r_{0}=0$, the Hayward black hole reduces to the Schwarzschild black hole. In the absence of the island, the entanglement entropy is the generalized entropy (8).

Let us calculate the entanglement entropy of the nonextremal Hayward black hole with an island. Extremizing 
condition of the generalized entropy (10) for the metric (37) with respect to $t_{a}$ is given as

$$
\frac{\partial S_{\mathrm{gen}}}{\partial t_{a}}=\frac{c \kappa_{H} \sinh \kappa_{H}\left(t_{a}-t_{b}\right)}{3\left[\cosh \kappa_{H}\left(t_{a}-t_{b}\right)-\cosh \kappa_{H}\left(r_{*}(a)-r_{*}(b)\right)\right]}=0
$$

in the late time approximation of $t_{a}+t_{b} \rightarrow \infty$, and thus, one can find $t_{a}=t_{b}$. In Eq. (39), the surface gravity is defined by

$\kappa_{H}=\frac{\left(r_{+}-r_{-}\right)\left(r_{+}-r_{0}\right)}{2\left(r_{+}^{3}-r_{+} r_{-} r_{0}\right)}$,

and the boundaries of the island and the radiation region in terms of the tortoise coordinates are written as

$$
\begin{aligned}
r_{*}(a)= & r_{+}+\frac{r_{+}^{3}-r_{+} r_{-} r_{0}}{\left(r_{+}-r_{-}\right)\left(r_{+}-r_{0}\right)} \log \left(\frac{a-r_{+}}{r_{+}}\right), \\
r_{*}(b)= & b+\frac{r_{+}^{3}-r_{+} r_{-} r_{0}}{\left(r_{+}-r_{-}\right)\left(r_{+}-r_{0}\right)} \log \left(\frac{b-r_{+}}{r_{+}}\right) \\
& -\frac{r_{-}^{3}-r_{+} r_{-} r_{0}}{\left(r_{+}-r_{-}\right)\left(r_{-}-r_{0}\right)} \log \left(\frac{b-r_{-}}{r_{+}}\right) \\
& +\frac{r_{0}^{3}-r_{+} r_{-} r_{0}}{\left(r_{+}-r_{0}\right)\left(r_{-}-r_{0}\right)} \log \left(\frac{b-r_{0}}{r_{+}}\right) .
\end{aligned}
$$

Next, the extremum condition with respect to $a$ is given as

$$
\begin{aligned}
\frac{\partial S_{\mathrm{gen}}}{\partial a}= & \frac{4 \pi r_{+}}{G_{N}}-\frac{c r_{+}}{r_{+}^{2}-r_{-} r_{0}}+\frac{c}{3}\left(\frac{1}{r_{+}-r_{-}}+\frac{1}{r_{+}-r_{0}}\right. \\
& \left.\times-\frac{1}{\sqrt{r_{+}\left(a-r_{+}\right)}} e^{\kappa_{H}\left(r_{+}-r_{*}(b)\right)}\right)=0,
\end{aligned}
$$

where $t_{a}=t_{b}$ was used with the late time approximation $t_{a}+t_{b} \rightarrow \infty$. From Eq. (43), the boundary of the island is determined as

$a \simeq r_{+}+\frac{\left(c G_{N}\right)^{2}}{144 \pi^{2} r_{+}^{3}} e^{2 \kappa_{H}\left(r_{+}-r_{*}(b)\right)}+\mathcal{O}\left(G_{N}^{3}\right)$.

Hence, the entanglement entropy of the non-extremal Hayward black hole with an island is obtained as entanglement entropy (45) in the leading order. The Page time is the same form as Eq. (26).

\subsection{Extremal Hayward black hole}

For $M=\left(3 \sqrt{3} / 4 G_{N}\right) \ell$ and $r_{0}=(-\sqrt{3} / 2) \ell$, the horizons are degenerate as $r_{+}=r_{-}=r_{h}=\sqrt{3} \ell$, and so the metric function for the extremal Hayward black hole is given as

$f(r)=\frac{\left(r-r_{h}\right)^{2}\left(2 r+r_{h}\right)}{2 r^{3}+r_{h}^{3}}$

where $r_{h}=(4 / 3) G_{N} M$.

By using Eq. (12), the entanglement entropy of the extremal Hayward black hole without an island is calculated as

$S=S_{\text {gen }} \approx \frac{c}{3} \log t_{b}$,

where we ignored the other finite terms in late times and used the fact that $f(0)=1$ in Eq. (46).

We now compute the entanglement entropy of the extremal Hayward black hole with an island by using Eq. (15). The extremization condition of the entanglement entropy (15) with respect to $t_{a}$ is

$\frac{\partial S_{\text {gen }}}{\partial t_{a}}=\frac{c\left(t_{a}-t_{b}\right)}{3\left[\left(t_{a}-t_{b}\right)^{2}-\left(r_{*}(a)-r_{*}(b)\right)^{2}\right]}=0$,

and thus, $t_{a}=t_{b}$ is found without the late time approximation. In Eq. (48), the tortoise coordinates for the boundaries are given as

$$
\begin{aligned}
r_{*}(a)= & r_{h}-\frac{r_{h}^{2}}{a-r_{h}}, \\
r_{*}(b)= & b-\frac{r_{h}^{2}}{b-r_{h}}+\frac{4 r_{h}}{3} \log \left(\frac{2\left(b-r_{h}\right)}{r_{h}}\right)+\frac{r_{h}}{6} \\
& \times \log \left(\frac{2 b+r_{h}}{r_{h}}\right) .
\end{aligned}
$$

Next, the derivative of the generalized entropy (15) with respect to $a$ is found to be

$$
\begin{aligned}
S_{I}= & \frac{2 \pi r_{+}^{2}}{G_{N}}+\frac{c}{6}\left[\log \left(\frac{16\left(r_{+}^{3}-r_{+} r_{-} r_{0}\right)^{3}\left(b-r_{+}\right)^{2}\left(b-r_{-}\right)\left(b-r_{0}\right)}{\left(b^{3}-r_{+} r_{-} r_{0}\right)\left(r_{+}-r_{-}\right)^{3}\left(r_{+}-r_{0}\right)^{3}}\right)-\frac{r_{-}^{3}-r_{+} r_{-} r_{0}}{r_{+}^{3}-r_{+} r_{-} r_{0}} \log \left(\frac{b-r_{-}}{r_{+}}\right)+\frac{r_{0}^{3}-r_{+} r_{-} r_{0}}{r_{+}^{3}-r_{+} r_{-} r_{0}}\right. \\
& \left.\times \log \left(\frac{b-r_{0}}{r_{+}}\right)+\frac{\left(r_{+}-r_{-}\right)\left(r_{+}-r_{0}\right)}{r_{+}^{3}-r_{+} r_{-} r_{0}}\left(b-r_{+}\right)\right] .
\end{aligned}
$$

For $r_{-}=r_{0}=0$, Eq. (45) exactly reduces to the entanglement entropy of the Schwarzschild black hole (25) in Ref. [20]. In addition, the Page time can be obtained at which the growing entanglement entropy (8) changes into the constant

$$
\frac{\partial S_{\mathrm{gen}}}{\partial a}=\frac{2 \pi r_{h}}{G_{N}}-\frac{c}{6\left(a-r_{h}\right)}-\frac{8 c}{9 r_{h}}+\frac{c}{3 r_{h}^{2}} r_{*}(b)=0 .
$$


If the island boundary is assumed to be very close to the horizon, $a \sim r_{+}$, then the boundary of the island is approximately determined as

$a \simeq r_{h}+\frac{c G_{N}}{12 \pi r_{h}}+\frac{\left(c G_{N}\right)^{2}\left(8 r_{h}-3 r_{*}(b)\right)}{216 \pi^{2} r_{h}^{4}}+\mathcal{O}\left(G_{N}^{3}\right)$

Thus, from Eq. (1), the entanglement entropy of the extremal Hayward black hole with an island is finally obtained as

$S_{\mathrm{I}}=\frac{\pi r_{h}^{2}}{G_{N}}+\frac{c}{12} \log \left(\frac{144 \pi^{2} r_{h}^{8}\left(b-r_{h}\right)^{2}\left(2 b+r_{h}\right)}{\left(c G_{N}\right)^{2}\left(2 b^{3}+r_{h}^{3}\right)}\right)$.

The extremal entanglement entropy (53) cannot be obtained by taking the extremal limit of Eq. (45).

Now, the Page time can be obtained at which the growing entanglement entropy (47) changes into the constant entanglement entropy (53) as

$t_{\text {Page }}=\left(\frac{144 \pi^{2} r_{h}^{8}\left(b-r_{h}\right)^{2}\left(2 b+r_{h}\right)}{\left(c G_{N}\right)^{2}\left(2 b^{3}+r_{h}^{3}\right)}\right)^{1 / 4} e^{\frac{3}{c} S_{\mathrm{BH}}}$,

in which we take into account up to the sub-leading logarithmic term in Eq. (53), and $S_{\mathrm{BH}}=\left(\pi r_{+}^{2}\right) / G_{N}$ is the Bekenstein-Hawking entropy. In the large black hole but $b \gg r_{h}$, the Page time in the extremal black hole (54) appears to be longer than Eq. (26) in the non-extremal black hole.

Let us explicate some special properties of the Hayward black hole in calculations of the entanglement entropy in the island proposal. In general relativity, the Schwarzschild black hole is geodesically incomplete in the presence of a curvature singularity responsible for breakdown of classical and quantum predictabilities. Fortunately, the curvature singularity is protected by the event horizon so that internal structures of the black hole are of no relevance to outside observers. A curvature singularity also appears in the Reissner-Nordström black hole. In the same manner, the curvature singularity is enclosed by the event horizon. But the latter case provides an extremal limit in contrast to the Schwarzschild black hole case.

In the island prescription, a curvature singularity inside the horizon may affect the calculation of the entanglement entropy of extremal black holes. Let us now mention what it happens in the extremal Reissner-Nordström black hole. In the calculations of the generalized entropy before the Page time in Fig. 2a, the entanglement entropy in the radiation region was not well-defined because of the curvature singularity at $b_{0}=\left(t_{0}, 0\right)$. Explicitly, as seen from Eq. (12), the lapse function is divergent, i.e., $f(0)=\infty$, which makes the generalized entropy divergent whereas $f(0)$ is finite in the Hayward black hole. Recall that a regularization of curvature singularity was unnecessary in the non-extremal case in Fig. 1a because the boundaries of the entanglement wedges never touch the curvature singularity. Usual islands or their boundaries were always positioned in a finite curvature region. On the other hand, after the Page time, the internal structure of the extremal Reissner-Nordström black hole also plays an important role. In Fig. 2b, the left boundary of the island necessarily ends at the origin; in other words, the timelike curvature singularity encounters the boundary of the spacelike island inside the horizon. In contrast to usual islands in a finite curvature region, the island in the extremal ReissnerNordström black hole encounters the curvature singularity. After the Page time, we have to consider the island contribution to the entanglement entropy for radiation but we do not understand how to handle the entanglement entropy formula when interacts between the particles on the left boundary of the island and the infinite curvature.

Fortunately, the Hayward black hole is asymptotically flat and everywhere regular. Especially, it has a de Sitter core: $f(r) \approx 1-r^{2} / \ell^{2}$. Moreover, it has two horizons like the Reissner-Nordström black hole. In order to study the entanglement entropy in the extremal case of a certain black hole with two horizons, it would be nice to consider a regular black hole such as the Hayward black hole. Before the Page time, in the extremal Hayward black hole, we can get the finite entanglement entropy (47) because the lapse function is finite, that is, $f(0)=1$. This finite result is different from the divergent entanglement entropy (12) of the extremal ReissnerNordström black hole with a singularity. After the Page time, we also obtained the entanglement entropy (53) without encountering complicated issue on interactions between the particles on the boundary of the island and the curvature singularity. These two features before and after the Page times are certainly advantages of the Hayward black hole.

At first sight, one might be tempting to regard the geometry of the extremal Reissner-Nordström black hole simply as the $A d S_{2} \times S^{2}$ so that the spacetime might be regular like the Jackiw-Teitelboim model in Ref. [12]. However, the extremal Reissner-Nordström black hole reduces $A d S_{2} \times S^{2}$ only near the horizon. From the full geometrical view-point, the curvature singularity still exists inside the horizon and affects calculations of the entanglement entropy of the extremal black hole in the island proposal. The whole geometry of the extremal Reissner-Nordström black hole is different from that of Jackiw-Teitelboim model in spite of providing almost the same entropy results. Consequently, in the island proposal, the Hayward black hole is appropriate to study the entanglement entropy of an extremal black hole.

\section{Conclusion and discussion}

In conclusion, we investigated the entanglement entropy of the Reissner-Nordström black hole and the Hayward black 
hole for the non-extremal and extremal cases. In our calculations, the eternal black holes with an island were assumed to be a priori in thermal equilibrium with a heat bath of an arbitrary temperature. Taking the on-shell condition, we obtained the generalized entropy and then found the entanglement entropy for each case. In particular, the island rule provided the well-defined fine-grained entropy even in the extremal black hole as long as the curvature is finite.

In connection with a heat bath of an arbitrary temperature, the present off-shell formulation of the entropy actually rests upon previous investigations calculating the statistical entropy of a scalar field on a classical black-hole background $[32,33]$. The thermal gas is assumed to be in thermal equilibrium with a thermal bath of an arbitrary temperature. The classical geometry and the thermal bath are treated independently because the thermal effect is intrinsically of no relevance to the classical geometry. After calculations of the statistical entropy for the arbitrary temperature, the temperature is finally set by the Hawking temperature, which results in the area law of the entropy.

Now we make a couple of comments. First, one of the distinctions of the Reissner-Nordström black hole from the Schwarzschild black hole is the appearance of the inner horizon. The Reissner-Nordström black hole appears to be unstable under small perturbations due to the mass inflation at the inner horizon [38]; however, we did not consider the mass inflation which is also responsible for the strong cosmic censorship. In our work, the eternal black holes were just assumed in order to figure out how the island rule can be applied to asymptotically flat black holes with inner and outer horizons. Second, in our calculations for the extremal Reissner-Nordström black hole with an island, the left-boundary of the island reached the singularity, which would be beyond the semi-classical approximations in that region. Of course, this issue does not appear in the Hayward black hole regular at the origin, but it needs some further investigations. Finally, the island formula was first derived from black holes in anti-de Sitter spacetime coupled to a nongravitational bath based on assumptions about factorization of the Hilbert space. Therefore, the Page curve in asymptotically flat spacetime may fail in the sense that information is always available outside a black hole in flat space even before the Page time [39,40]. Even weak effects from a gravitational bath can provide different answers from local theories [41]. It means that concrete theoretical investigations are needed in such a way that the island rule can be well applied to asymptotically flat spacetimes.

Acknowledgements We would like to thank Hwajin Eom, Jeongwon Ho, Sojeong Jung, and Sang-Heon Yi for helpful and stimulating discussions. We also wish to thank Suvrat Raju for helpful comments on heat baths. This research was supported by Basic Science Research Program through the National Research Foundation of Korea(NRF) funded by the Korea government(MSIP) (Grant no. 2017R1A2B2006159) and the Ministry of Education through the Center for Quantum Spacetime (CQUeST) of Sogang University (NRF-2020R1A6A1A03047877).

Data Availability Statement This manuscript has no associated data or the data will not be deposited. [Authors' comment: There is no data for deposit.]

Open Access This article is licensed under a Creative Commons Attribution 4.0 International License, which permits use, sharing, adaptation, distribution and reproduction in any medium or format, as long as you give appropriate credit to the original author(s) and the source, provide a link to the Creative Commons licence, and indicate if changes were made. The images or other third party material in this article are included in the article's Creative Commons licence, unless indicated otherwise in a credit line to the material. If material is not included in the article's Creative Commons licence and your intended use is not permitted by statutory regulation or exceeds the permitted use, you will need to obtain permission directly from the copyright holder. To view a copy of this licence, visit http://creativecomm ons.org/licenses/by/4.0/.

Funded by $\mathrm{SCOAP}^{3}$.

\section{References}

1. S.W. Hawking, Particle creation by black holes. Commun. Math. Phys. 43, 199-220 (1975)

2. S.W. Hawking, Breakdown of predictability in gravitational collapse. Phys. Rev. D 14, 2460-2473 (1976)

3. W.G. Unruh, R.M. Wald, Information loss. Rep. Prog. Phys. 80, 092002 (2017). arXiv: 1703.02140

4. D.N. Page, Information in black hole radiation. Phys. Rev. Lett. 71, 3743-3746 (1993). arXiv:hep-th/9306083

5. S. Ryu, T. Takayanagi, Holographic derivation of entanglement entropy from AdS/CFT. Phys. Rev. Lett. 96, 181602 (2006). arXiv:hep-th/0603001

6. V.E. Hubeny, M. Rangamani, T. Takayanagi, A covariant holographic entanglement entropy proposal. JHEP 07, 062 (2007). arXiv:0705.0016

7. T. Barrella, X. Dong, S.A. Hartnoll, V.L. Martin, Holographic entanglement beyond classical gravity. JHEP 09, 109 (2013). arXiv: 1306.4682

8. T. Faulkner, A. Lewkowycz, J. Maldacena, Quantum corrections to holographic entanglement entropy. JHEP 11, 074 (2013). arXiv: 1307.2892

9. N. Engelhardt, A.C. Wall, Quantum extremal surfaces: holographic entanglement entropy beyond the classical regime. JHEP 01, 073 (2015). arXiv: 1408.3203

10. G. Penington, Entanglement wedge reconstruction and the information paradox. JHEP 09, 002 (2020). arXiv: 1905.08255

11. A. Almheiri, N. Engelhardt, D. Marolf, H. Maxfield, The entropy of bulk quantum fields and the entanglement wedge of an evaporating black hole. JHEP 12, 063 (2019). arXiv: 1905.08762

12. A. Almheiri, R. Mahajan, J. Maldacena, Islands outside the horizon, arXiv: 1910.11077

13. A. Almheiri, R. Mahajan, J. Maldacena, Y. Zhao, The Page curve of Hawking radiation from semiclassical geometry. JHEP 03, 149 (2020). arXiv: 1908.10996

14. G. Penington, S.H. Shenker, D. Stanford, Z. Yang, Replica wormholes and the black hole interior 1911, 1 (1977)

15. A. Almheiri, T. Hartman, J. Maldacena, E. Shaghoulian, A. Tajdini, Replica wormholes and the entropy of hawking radiation. JHEP $\mathbf{0 5}$, 01 (2020). arXiv:1911.12333

16. L. Bombelli, R.K. Koul, J. Lee, R.D. Sorkin, A quantum source of entropy for black holes. Phys. Rev. D 34, 373-383 (1986) 
17. M. Srednicki, Entropy and area. Phys. Rev. Lett. 71, 666-669 (1993). arXiv:hep-th/9303048

18. L. Susskind, J. Uglum, Black hole entropy in canonical quantum gravity and superstring theory. Phys. Rev. D 50, 2700-2711 (1994). arXiv:hep-th/9401070

19. A. Almheiri, R. Mahajan, J.E. Santos, Entanglement islands in higher dimensions. SciPost Phys. 9, 001 (2020). arXiv:1911.09666

20. K. Hashimoto, N. Iizuka, Y. Matsuo, Islands in Schwarzschild black holes. JHEP 06, 085 (2020). arXiv:2004.05863

21. T. Anegawa, N. Iizuka, Notes on islands in asymptotically flat $2 \mathrm{~d}$ dilaton black holes. JHEP 07, 036 (2020). arXiv:2004.01601

22. D. Bak, C. Kim, S.-H. Yi, J. Yoon, Unitarity of entanglement and islands in two-sided Janus black holes. JHEP 01, 155 (2021). arXiv:2006.11717

23. Y. Ling, Y. Liu, Z.-Y. Xian, Island in Charged Black Holes. arXiv:2010.00037

24. G.K. Karananas, A. Kehagias, J. Taskas, Islands in linear dilaton black holes. arXiv:2101.00024

25. X. Wang, R. Li, J. Wang, Quantifying islands and Page curves of Reissner-Nordstrl"om black holes for resolving information paradox. arXiv:2101.06867

26. C. Krishnan, V. Patil, J. Pereira, Page curve and the information paradox in flat space. arXiv:2005.02993

27. A. Almheiri, T. Hartman, J. Maldacena, E. Shaghoulian and A. Tajdini, The entropy of Hawking radiation. arXiv:2006.06872

28. C. Krishnan, Critical islands. JHEP 01, 179 (2021). arXiv:2007.06551

29. H. Geng, Y. Nomura, H.-Y. Sun, An information paradox and its resolution in de Sitter holography. arXiv:2103.07477

30. K. Ghosh, C. Krishnan, Dirichlet baths and the not-so-fine-grained page curve. arXiv:2103.17253
31. S.W. Hawking, G.T. Horowitz, S.F. Ross, Entropy, area, and black hole pairs. Phys. Rev. D 51, 4302-4314 (1995). arXiv:gr-qc/9409013

32. G. 't Hooft, On the quantum structure of a black hole. Nucl. Phys. B 256, 727-745 (1985)

33. J.-G. Demers, R. Lafrance, R.C. Myers, Black hole entropy without brick walls. Phys. Rev. D 52, 2245-2253 (1995). arXiv:gr-qc/9503003

34. A. Strominger, C. Vafa, Microscopic origin of the BekensteinHawking entropy. Phys. Lett. B 379, 99-104 (1996). arXiv:hep-th/9601029

35. S.M. Carroll, M.C. Johnson, L. Randall, Extremal limits and black hole entropy. JHEP 11, 109 (2009). arXiv:0901.0931

36. S.A. Hayward, Formation and evaporation of regular black holes. Phys. Rev. Lett. 96, 031103 (2006). arXiv:gr-qc/0506126

37. P. Calabrese, J.L. Cardy, Entanglement entropy and quantum field theory. J. Stat. Mech. 0406, P06002 (2004). arXiv:hep-th/0405152

38. E. Poisson, W. Israel, Internal structure of black holes. Phys. Rev. D 41, 1796-1809 (1990)

39. A. Laddha, S.G. Prabhu, S. Raju, P. Shrivastava, The holographic nature of null infinity. SciPost Phys. 10, 041 (2021). arXiv:2002.02448

40. S. Raju, Lessons from the Information Paradox. arXiv:2012.05770

41. H. Geng, A. Karch, C. Perez-Pardavila, S. Raju, L. Randall, M. Riojas et al., Information Transfer with a Gravitating Bath. arXiv:2012.04671 